\title{
Tourette syndrome research in Europe has entered a new era of collaboration
}

\author{
Pieter J. Hoekstra $\cdot$ Andrea Dietrich
}

Published online: 4 February 2015

(C) Springer-Verlag Berlin Heidelberg 2015

Tourette syndrome (TS) is a fascinating disorder, being at the cross-roads of a neurological movement disorder and a neuropsychiatric disorder; in many cases, the movements and vocalizations are not entirely beyond a patient's voluntary control and some of its features share similarities with the symptoms of obsessive-compulsive disorder and/or the stereotyped movements associated with autism spectrum disorder. An interesting feature is the often intricate link between the type and pattern of an affected individual's tics with his or her environment. A classic example is that tics may be 'contagious': many persons with TS have had the experience at TS patient association meetings of a tendency to adopt tics they see in others. Also, many affected individuals can distinguish between situations that clearly tend to trigger their tics versus other situations in which they may be largely tic-free. The difference in presentation between the two settings can often be striking and this may confuse parents and teachers, leaving them sometimes wonder to what extent the so variable behavior should be seen as a 'disorder'. That is exactly why explaining to families and school personnel the changing nature of tic symptoms over time and across settings as a hallmark of TS is such an important element of treatment.

As is the case with most neuropsychiatric disorders, our understanding of the etiology and pathophysiology of TS is far from complete. Indeed the history of the prevailing views of TS over the past century is almost as fascinating as the disorder. An excellent read on the changing views on TS is provided in Howard Kushner's book A cursing brain?

P. J. Hoekstra $(\bowtie) \cdot$ A. Dietrich

Department of Child and Adolescent Psychiatry Groningen,

University of Groningen, University Medical Center Groningen,

Groningen, The Netherlands

e-mail: p.hoekstra@accare.nl
[1]. Especially the section reviewing the work of Margaret Mahler on tics in children is as far away from modern views as possible, in that she indicated that it were mothers who interfered with their children's motor expression, as a consequence of which the children were unable to assert normal control over their motor activities.

Nowadays, TS is viewed as a largely hereditary disorder, with genes interacting with environmental factors such as pregnancy and delivery complications, psychosocial stress later in life and possibly infections [2]. Since long, TS has been associated with fronto-striatal-thalamic abnormalities. The fronto-striatal circuit, which comprises reciprocal connections among the striatum, thalamus, and prefrontal areas, is critical to cognitive control. Five basal ganglia loops have been outlined [3], which when dysfunctional, have motor and/or cognitive implications, with the sensory-motor loop (sensory-motor and supplementary motor cortex; dorsolateral putamen; ventrolateral globus pallidus and ventrolateral thalamus) primarily being affected in TS [4]. Functional structural and diffusion magnetic resonance imaging (MRI) have also pointed to immature and anomalous patterns of functional and structural connectivity in resting state networks such as the default mode network [5, 6]. Resting-state functional connectivity MRI has implicated deviation of connectivity in the fronto-parietal network, important for online adaptive control [7, 8]. Although TS has classically been linked to dopamine dysfunctioning, recent studies have pointed to the involvement of other neurotransmitter systems, most notably glutamate (see [8] for a review).

At least five current major limitations hamper the field of brain imaging studies in TS: (1) there has been a strong focus on TS in adults with underrepresentation of studies in children and adolescents with TS; (2) there is a lack of longitudinal MRI studies; (3) the potentially confounding 
effects of comorbidity have insufficiently been taken into account; (4) studies have mostly used very small sample sizes; and (5) metabolic imaging studies have been extremely scarce so far. Regarding the importance of developmental stage, indeed, the review by Gerard and Peterson [9] has pointed out the major divergence in findings between adult and child studies of TS. For example, reduced caudate volume in children with TS has been found while there is increased caudate volume in adults with TS. Only longitudinal studies, beginning in childhood, of the course of TS allow compensatory changes and markers of illness persistence to be identified.

Scientific progress has been slow, compared to other disorders such as ADHD and autism spectrum disorders, largely due to a lack of collaborative large-scale studies. Luckily, in Europe and world-wide, TS has entered a new era of collaboration. At least three major collaborative efforts are ongoing within Europe. This issue of European Child and Adolescent Psychiatry describes the set-up of the Tourette International Collaborative Genetics (TIC Genetics) study, a USA-European collaboration aimed at identifying genes associated with TS (see the article "The Tourette International Collaborative Genetics (TIC Genetics) study, finding the genes causing Tourette syndrome: objectives and methods" in this issue). Other ongoing studies include the European Multicenter Tics in Children Studies (EMTICS), investigating the interplay between environmental triggers, the immune system, and genetic factors in association with tic onset and exacerbations [10] and TSEUROTRAIN, a major collaborative interdisciplinary effort investigating genetics, neuroimaging, and animal models of TS. Collaborative studies are of eminent importance to advance progress in the field of TS. These are indeed fascinating times for improving our understanding of TS, with the ultimate goal of developing better treatments to affected children and their families.

\section{References}

1. Kushner HI (1999) A cursing brain? The histories of Tourette Syndrome. Harvard University Press, Cambridge

2. Hoekstra PJ, Dietrich A, Edwards MJ, Elamin I, Martino D (2013) Environmental factors in Tourette syndrome. Neurosci Biobehav Rev 37(6):1040-1049

3. Leisman G, Melillo R (2013) The basal ganglia: motor and cognitive relationships in a clinical neurobehavioral context. Rev Neurosci 24(1):9-25

4. Makki MI, Govindan RM, Wilson BJ, Behen ME, Chugani HT (2009) Altered fronto-striato-thalamic connectivity in children with Tourette syndrome assessed with diffusion tensor MRI and probabilistic fiber tracking. J Child Neurol 24(6):669-678

5. Neuner I, Werner CJ, Arrubla J, Stöcker T, Ehlen C, Wegener HP, Schneider F, Shah NJ (2014) Imaging the where and when of tic generation and resting state networks in adult Tourette patients. Front Hum Neurosci 28(8):362

6. Worbe Y, Marrakchi-Kacem L, Lecomte S, Valabregue R, Poupon F, Guevara P, Tucholka A, Mangin JF, Vidailhet M, Lehericy S, Hartmann A, Poupon C (2014) Altered structural connectivity of cortico-striato-pallido-thalamic networks in Gilles de la Tourette syndrome. Brain. doi:10.1093/brain/awu311

7. Church JA, Fair DA, Dosenbach NU, Cohen AL, Miezin FM, Petersen SE, Schlaggar BL (2009) Control networks in paediatric Tourette syndrome show immature and anomalous patterns of functional connectivity. Brain 132(Pt 1):225-238

8. Paschou P, Fernandez TV, Sharp F, Heiman GA, Hoekstra PJ (2013) Genetic susceptibility and neurotransmitters in Tourette syndrome. Int Rev Neurobiol 112:155-177

9. Gerard E, Peterson BS (2003) Developmental processes and brain imaging studies in Tourette syndrome. J Psychosom Res 55(1):13-22

10. Roessner V, Hoekstra PJ (2013) European Multicenter Tics in Children Studies (EMTICS): exploring the onset and course of tic disorders. Eur Child Adolesc Psychiatry 22(7):451-452 